\title{
ARTICLE
}

Clinical Research

\section{Examining differences between overweight women and men in 12-month weight loss study comparing healthy low-carbohydrate vs. low-fat diets}

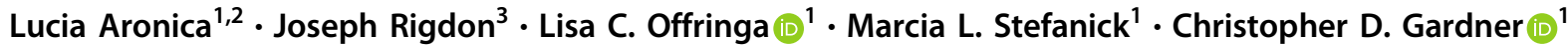

Received: 1 October 2019 / Revised: 25 September 2020 / Accepted: 29 October 2020 / Published online: 14 November 2020

(c) The Author(s), under exclusive licence to Springer Nature Limited 2020. This article is published with open access

\begin{abstract}
Background/objectives Biological sex factors and sociocultural gender norms affect the physiology and behavior of weight loss. However, most diet intervention studies do not report outcomes by sex, thereby impeding reproducibility. The objectives of this study were to compare 12-month changes in body weight and composition in groups defined by diet and sex, and adherence to a healthy low carbohydrate (HLC) vs. healthy low fat (HLF) diet.

Participants/methods This was a secondary analysis of the DIETFITS trial, in which 609 overweight/obese nondiabetic participants (age, 18-50 years) were randomized to a 12-month HLC $(n=304)$ or HLF $(n=305)$ diet. Our first aim concerned comparisons in 12-month changes in weight, fat mass, and lean mass by group with appropriate adjustment for potential confounders. The second aim was to assess whether or not adherence differed by diet-sex group (HLC women $n=$ 179, HLC men $n=125$, HLF women $n=167$, HLF men $n=138$ ).

Results 12 -month changes in weight $(p<0.001)$ were different by group. HLC produced significantly greater weight loss, as well as greater loss of both fat mass and lean mass, than HLF among men $[-2.98 \mathrm{~kg}(-4.47,-1.50) ; P<0.001]$, but not among women. Men were more adherent to HLC than women $(p=0.02)$. Weight loss estimates within group remained similar after adjusting for adherence, suggesting adherence was not a mediator.

Conclusions By reporting outcomes by sex significant weight loss differences were identified between HLC and HLF, which were not recognized in the original primary analysis. These findings highlight the need to consider sex in the design, analysis, and reporting of diet trials.
\end{abstract}

These authors contributed equally: Marcia L. Stefanick, Christopher D. Gardner

Supplementary information The online version of this article (https:// doi.org/10.1038/s41366-020-00708-y) contains supplementary material, which is available to authorized users.

Christopher D. Gardner

cgardner@stanford.edu

1 Department of Medicine, Stanford Prevention Research Center, Stanford University School of Medicine, Stanford, CA, USA

2 Department of Nutritional Sciences, University of Vienna, Vienna, Austria

3 Quantitative Sciences Unit, Stanford University School of Medicine, Stanford, CA, USA

\section{Introduction}

As precision medicine is gaining traction, the NIH has highlighted the need to consider sex as a biological variable (SABV) in animal and human research [1-3]. Diet interventions remain the primary strategy in obesity management; yet, their effectiveness is highly variable in the general population and among research participants [4]. Some of this variability reflects biological "sex" differences, such as body composition and metabolism, and some reflects sociocultural "gender" factors, which can influence food behaviors, such as dietary preferences and adherence. In terms of biological sex differences, men tend to lose more weight on a diet intervention because, on average, they have greater body size, higher muscle-to-fat mass ratio, and higher resting and total energy expenditure. They also tend to accumulate more intra-abdominal fat than women, which is associated with higher risk of metabolic syndrome (MetS) and better response to low-carbohydrate diets [5-9]. 
In terms of behavioral gender differences, women are more likely to attempt weight loss multiple times and join weight loss studies than men [10-12]. They also express a higher preference for low-fat (LF) products and a higher concern towards high-fat foods [12], which might make it easier for them to adhere to a LF diet.

Because most weight loss trials have a much higher representation of women than men, only a few trials have had sufficient power to compare the effects of caloric restriction on weight loss or body composition changes in women vs. men $[13,14]$, particularly with respect to effects of diets of different macronutrient composition on total weight loss [9, 15-19] or fat and/or lean mass changes [9, 15, 17, 20-23]. In addition, most of these trials had small sample sizes (fewer than 100 participants) and/or were of short duration (less than 6 months). While these studies generally reported greater absolute weight loss for women than for men, these differences were often not significant when adjusted for baseline weight [24, 25]. In a previous study, when sex was not considered, adherence was comparable on a low-fat vs. low-carb diet, but was associated with greater weight loss only for the low carb group [26].

To address this gap in the literature, a secondary analysis of the Diet Intervention Examining The Factors Interacting with Treatment Success trial (DIETFITS) was conducted. DIETFITS involved 609 overweight/obese non-diabetic participants (age, 18-50 years) that were randomized to a 12-month healthy low-carbohydrate (HLC) $(n=304)$ or healthy LF (HLF) $(n=305)$ diet [27]. The first aim of this study was to compare 12-month changes in body weight, fat mass, and lean mass in women and men assigned to HLC or HLF (HLC women $n=179$, HLC men $n=125$, HLF women $n=167$, HLF men $n=138$ ). The second aim was to assess whether or not adherence differed by dietsex group.

\section{Participants and methods}

\section{Study design and participants}

The original DIETFITS trial was a single-site, parallelgroup, randomized trial of 609 overweight/obese women ( $n$ $=346)$ and men $(n=263)$ conducted at the Stanford Prevention Research Center from January 2013 to May 2016 and was designed to test whether baseline genetic or metabolic factors would explain any of the differential weight loss for participants assigned to HLF vs. HLC [27]. The detailed primary study protocol has been reported elsewhere [28]. Briefly, participants were 609 generally healthy adults assigned to HLC ( $n=179$ women, $n=125$ men) or HLF ( $n=167$ women, $n=138$ men) aged $18-50$ years, with body mass indices $28-40 \mathrm{~kg} / \mathrm{m}^{2}$. Exclusion criteria included uncontrolled metabolic disease or hypertension; pregnancy or lactation; diabetes; cancer; cardiovascular, renal or liver disease; or use of medications expected to affect weight. Randomization to HLF or HLC was performed using an allocation sequence determined by computerized random-number generation [27]. The weight loss intervention involved a 12-month protocol of 22 smallgroup educational sessions focused on three central components for both HLC and HLF [28]. During the first 8 weeks of limbo phase, participants were instructed to cut back on fat or carbohydrate intake progressively until they achieved a daily intake of no more than $20 \mathrm{~g}$ of carbohydrate (HLC) or fat (HLF). During the titrate phase, participants were instructed to increase their fat or carbohydrate intake slowly, by 5-15 g each week, until they achieved a comfortable maintenance level. The goal of this phase was to find the lowest intake of fat or carbohydrates that each participant could maintain for the 12-month intervention period. The third diet intervention component was high quality, for which both groups received similar instructions to focus on home-cooked whole foods, maximize fresh, seasonal vegetables, lean, grass-fed meats, and eliminate or minimize processed foods with added sugar, refined white flour and trans-fats. All study participants provided written informed consent about the study procedures. The study was registered on clinicaltrials.gov with the identifier: NCT01826591.

\section{Outcome measurements}

\section{Weight, height, body mass index, and fat and lean body mass}

Body weight was measured at baseline, and months 3, 6, and 12. Weight was measured in light clothing to the nearest $0.1 \mathrm{~kg}$ using a calibrated clinical scale. Height was measured to the nearest $0.1 \mathrm{~cm}$ using a Seca wallmounted stadiometer. Body Mass Index (BMI) was calculated as the weight in $\mathrm{kg}$ divided by height in meters squared.

Body composition was assessed by dual-energy x-ray absorptiometry (DEXA) at baseline and months 6 and 12 [28]. Access to this was made available only after the first $n=78$ participants had been enrolled (i.e., none of the first 78 participants had a baseline measurement taken); from that point on DEXA was assessed in $87 \%$ of the remaining participants ( $n=276$ women, $n=190$ men).

\section{Dietary intake and adherence}

Dietary intake was assessed at baseline and months 3, 6, and 12 using three unannounced 24-h multiple-pass recall interviews ( 2 weekdays, 1 weekend day) [29]. Adherence 
was measured via a weight-adjusted standardized adherence (WASA) score based on the deviation score (DS) between the Limbo phase macronutrient goal ( $20 \mathrm{~g}$ of fat on HLF or carbohydrate on HLC) and participants reported dietary intake. A WASA score was calculated for each participant by diet at each time point $(3,6$, or 12 months) with available dietary data as follows: (a) up to three recalls per data collection time point were averaged as an estimate of macronutrient consumption, e.g., $50 \mathrm{~g}$ carbohydrates; (b) deviation from the target of $20 \mathrm{~g}$ of carbohydrates (or fat) was calculated as $20-50=-30 \mathrm{~g}$; (c) the DS was equal to the deviation divided by baseline weight in $\mathrm{kg}$, e.g., $-30 /$ $60 \mathrm{~kg}$ yields DS of $-1 / 2$; (d) DS were normalized (Z-score) within diet and timepoint by subtracting the mean and dividing by the standard deviation, e.g., if the mean (standard deviation) DS of HLC at 3 months was 0.5 [4], the Zscore at 3 months would be $(-1 / 2-0.5) / 4=-1 / 4$; and, (e) WASA for each participant was calculated as the average of available $Z$-scores at 3, 6, and 12 months. A WASA score of 0 reflects an average degree of adherence relative to all groups; positive and negative scores reflect better and worse adherence than average across all groups, respectively. A 12-month dietary WASA for each participant was then calculated as the average of any available WASA scores from the three post-randomization time points $(3,6$, and 12 months).

\section{Food choice questionnaires}

To assess attitudes toward dietary carbohydrates or fat, we selected two questions from the battery of psychosocial questionnaires administered in the study that were adapted from a previously validated food choice questionnaire (FCQ) developed by Steptoe et al.: The first statement asked to express the degree of preference for LF food on a typical day [statement: "It is important to me that the food I eat on a typical day: Is low in Fat": possible answers: (1) Very important, (2) Moderately important, (3) A little important, (4) Not at all important]. The second statement asked to report the tendency to avoid foods high in refined carbohydrates [statement: "Particularly avoid foods with high carbohydrate content (e.g., bread, pasta, rice, etc.); possible answers: (1) Always, (2) Very Often, (3) Often, (4) Sometimes, (5) Rarely, (6) Never].

\section{Statistical analysis}

We tested three main hypotheses: (i) 12-month changes in key outcomes (weight, fat mass, and lean mass) were different by diet-sex group (HLC women, HLC men, HLF women, HLF men), (ii) adherence was different by diet-sex group, (iii) 12-month changes in key outcomes by diet-sex group were mediated by adherence.
Linear mixed effects models were used to address hypothesis (i). Linear mixed effects can flexibly model incomplete longitudinal data under a missing at random assumption. Models included fixed effects for diet-sex group, time, and their interaction, with additional fixed effects for the potential confounders of baseline weight and baseline percent fat, and included a random intercept term for participants. An $F$-test with Satterthwaite adjustment for denominator degrees of freedom was used to test the null hypothesis that 12-month changes are equivalent in diet-sex groups. T-tests with Kenward-Roger degrees of freedom adjustment were employed for the four pairwise comparisons of interest.

To address hypothesis (ii), WASA scores were modeled in a linear regression as a function of diet-sex group. An overall $F$-test was used to test the association, with $t$-tests for four pairwise comparisons of interest: HLC vs. HLF within women; HLC vs. HLF within men; men vs. women within HLF; and, men vs. women within HLC.

Hypothesis (iii) was tested using the same linear mixed model framework as hypothesis (i), with the addition of WASA as a fixed effect. Overall $F$-tests and pairwise $t$-tests (both with appropriate degrees of freedom adjustment) were again employed to test differences in 12-month outcomes. Relationships between adherence (WASA) and percent change in 12-month outcomes were also characterized via scatterplot and Spearman rank correlation.

All statistical tests were two-sided at significance level 0.05 . Given the exploratory nature of this secondary analysis, no adjustments for multiple testing were performed. All statistical analyses were carried out using $R$ version 3.6.1 [30] and code is available at https://github.com/ joerigdon/DIETFITS_Gender.

\section{Results}

\section{Baseline characteristics of the study population}

Among 609 participants originally randomized to HLC (women, $n=179$; men, $n=125$ ) or HLF (women, $n=167$; men, $n=138$ ), overall 12 -months retention, i.e., providing any data at 12 months, was $\sim 79 \%$ for each group, with no significant between-group differences. Mean age was about 38-40 years for all four groups. There were no statistically significant baseline differences in weight between HLC and HLF women or between HLC and HLF men (Table 1). Women weighed less than men, but all groups had similar BMI (about $33 \mathrm{~kg} / \mathrm{m}^{2}$ ). Participants who received DEXA measurement $(n=466)$ were heavier $(5 \pm 1.4 \mathrm{~kg})$ than those who did not $(n=143)$ (Supplementary Table S1). Men had a significantly higher MetS score and associated variables (i.e., triglycerides, HDL-C, insulin-30, fasting glucose and insulin, blood pressure) compared with women, which is in 
Table 1 Baseline demographics and anthropometric and metabolic variables.

\begin{tabular}{|c|c|c|c|c|c|}
\hline & $\begin{array}{l}\text { HLC women } \\
n=179\end{array}$ & $\begin{array}{l}\text { HLC men } \\
n=125\end{array}$ & $\begin{array}{l}\text { HLF women } \\
n=167\end{array}$ & $\begin{array}{l}\text { HLF men } \\
n=138\end{array}$ & $P$ value $^{1}$ \\
\hline Age (years) & $40.2( \pm 6.9)$ & $40.2( \pm 6.5)$ & $39.7( \pm 6.4)$ & $38.9( \pm 7.3)$ & 0.41 \\
\hline \multicolumn{6}{|l|}{ Education } \\
\hline High school & $48(26.8 \%)$ & $34(27.2 \%)$ & $41(24.6 \%)$ & $29(21.0 \%)$ & 0.85 \\
\hline College & $73(40.8 \%)$ & $45(36.0 \%)$ & $68(40.7 \%)$ & $59(42.8 \%)$ & \\
\hline Grad degree & $58(32.4 \%)$ & $45(36.0 \%)$ & $57(34.1 \%)$ & $50(36.2 \%)$ & \\
\hline Missing & $0(0.0 \%)$ & $1(0.8 \%)$ & $1(0.6 \%)$ & $0(0.0 \%)$ & \\
\hline \multicolumn{6}{|l|}{ Race/ethnicity } \\
\hline White & $111(62.0 \%)$ & $71(56.8 \%)$ & $91(54.5 \%)$ & $85(61.6 \%)$ & 0.068 \\
\hline Hispanic & $35(19.6 \%)$ & $26(20.8 \%)$ & $44(26.3 \%)$ & $23(16.7 \%)$ & \\
\hline Asian & $19(10.6 \%)$ & $11(8.8 \%)$ & $9(5.4 \%)$ & $21(15.2 \%)$ & \\
\hline African-American & $7(3.9 \%)$ & $6(4.8 \%)$ & $8(4.8 \%)$ & $2(1.4 \%)$ & \\
\hline AI/AN/PI & $0(0.0 \%)$ & $0(0.0 \%)$ & $2(1.2 \%)$ & $1(0.7 \%)$ & \\
\hline Other & $7(3.9 \%)$ & $11(8.8 \%)$ & $13(7.8 \%)$ & $6(4.3 \%)$ & \\
\hline Weight (kg) & $88.9( \pm 12.5)^{\mathrm{ab}}$ & $106.8( \pm 13.7)^{\mathrm{ac}}$ & $90.7( \pm 11.5)^{\mathrm{cd}}$ & $105.7( \pm 13.9)^{\mathrm{bd}}$ & $<0.0001$ \\
\hline BMI $\left(\mathrm{kg} / \mathrm{m}^{2}\right)$ & $32.9( \pm 3.4)$ & $33.8( \pm 3.4)$ & $33.3( \pm 3.4)$ & $33.5( \pm 3.4)$ & 0.16 \\
\hline Body fat $(\%)$ & $40.4( \pm 4.0)^{\mathrm{ab}}$ & $30.3( \pm 4.7)^{\mathrm{ac}}$ & $41.0( \pm 3.9)^{\mathrm{cd}}$ & $29.9( \pm 4.5)^{\mathrm{bd}}$ & $<0.0001$ \\
\hline Missing & $34(19.0 \%)$ & $32(25.6 \%)$ & $36(21.6 \%)$ & $41(29.7 \%)$ & \\
\hline Waist circumference $(\mathrm{cm})$ & $102.6( \pm 10.5)^{\mathrm{ab}}$ & $112.7( \pm 9.9)^{\mathrm{ac}}$ & $103.5( \pm 10.4)^{\mathrm{cd}}$ & $111.8( \pm 9.7)^{\mathrm{bd}}$ & $<0.0001$ \\
\hline Missing & $0(0 \%)$ & $2(1.6 \%)$ & $1(0.6 \%)$ & $2(1.4 \%)$ & \\
\hline LDL (mg/dL) & $111.6( \pm 26.3)$ & $117.1( \pm 25.6)$ & $109.1( \pm 29.0)$ & $114.8( \pm 32.1)$ & 0.056 \\
\hline Missing & $1(0.6 \%)$ & $0(0 \%)$ & $0(0 \%)$ & $0(0 \%)$ & \\
\hline $\mathrm{HDL}(\mathrm{mg} / \mathrm{dL})$ & $52.3( \pm 9.4)^{\mathrm{ab}}$ & $46.1( \pm 7.6)^{\mathrm{ac}}$ & $52.1( \pm 9.1)^{\mathrm{cd}}$ & $46.2( \pm 7.4)^{\mathrm{bd}}$ & $<0.0001$ \\
\hline Triglycerides (mg/dL) & $117.3( \pm 104.2)^{a b}$ & $143.4( \pm 66.3)$ & $114.9( \pm 66.0)^{\mathrm{cd}}$ & $145.2( \pm 74.0)^{\mathrm{bd}}$ & $<0.0001$ \\
\hline $\mathrm{SBP}(\mathrm{mmHg})$ & $120.2( \pm 12.6)^{\mathrm{ab}}$ & $126.7( \pm 11.0)^{\mathrm{ac}}$ & $118.6( \pm 11.9)^{\mathrm{cd}}$ & $127.9( \pm 11.4)^{\mathrm{bd}}$ & $<0.0001$ \\
\hline Missing & $1(0.6 \%)$ & $0(0 \%)$ & $2(1.2 \%)$ & $0(0 \%)$ & \\
\hline DBP & $79.7( \pm 7.8)^{\mathrm{ab}}$ & $83.3( \pm 7.3)^{\mathrm{ac}}$ & $78.8( \pm 6.9)^{\mathrm{cd}}$ & $83.6( \pm 7.0)^{\mathrm{bd}}$ & $<0.0001$ \\
\hline Missing & $1(0.6 \%)$ & $0(0 \%)$ & $2(1.2 \%)$ & $0(0 \%)$ & \\
\hline Fasting glucose (mg/dL) & $96.5( \pm 9.0)^{\mathrm{ab}}$ & $101.2( \pm 10.1)^{\mathrm{ac}}$ & $96.8( \pm 8.3)^{\mathrm{cd}}$ & $100.9( \pm 8.5)^{\mathrm{bd}}$ & $<0.0001$ \\
\hline Fasting insulin $(\mu \mathrm{IU} / \mathrm{mL})$ & $14.2( \pm 7.3)^{\mathrm{ab}}$ & $17.2( \pm 8.7)$ & $13.6( \pm 6.7)^{\mathrm{cd}}$ & $18.6( \pm 18.3)^{\mathrm{bd}}$ & $<0.0001$ \\
\hline Insulin-30 $(\mu \mathrm{IU} / \mathrm{mL})$ & $85.1( \pm 57.8)^{\mathrm{ab}}$ & $101.4( \pm 66.0)^{\mathrm{a}}$ & $88.5( \pm 66.5)$ & $103.1( \pm 68.1)^{\mathrm{b}}$ & 0.005 \\
\hline Missing & $0(0 \%)$ & $0(0 \%)$ & $2(1.2 \%)$ & $1(0.7 \%)$ & \\
\hline \multicolumn{6}{|l|}{ Metabolic syndrome } \\
\hline No & $121(67.6 \%)^{\mathrm{ab}}$ & $47(37.6 \%)^{\mathrm{ac}}$ & $110(65.9 \%)^{\mathrm{cd}}$ & $52(37.7 \%)^{\mathrm{bd}}$ & $<0.0001$ \\
\hline Yes & $58(32.4 \%)$ & $78(62.4 \%)$ & $57(34.1 \%)$ & $86(62.3 \%)$ & \\
\hline Respiratory exchange ratio & $0.86( \pm 0.06)$ & $0.87( \pm 0.06)$ & $0.85( \pm 0.06)$ & $0.87( \pm 0.07)$ & 0.087 \\
\hline Missing & $25(14.0 \%)$ & $15(12.0 \%)$ & $21(12.6 \%)$ & $20(14.5 \%)$ & \\
\hline $\begin{array}{l}\text { Resting energy } \\
\text { expenditure (kcal) }\end{array}$ & $1488.2( \pm 215.4)^{\mathrm{ab}}$ & $\begin{array}{l}1825.3 \\
( \pm 275.0)^{\mathrm{ac}}\end{array}$ & $1500.8( \pm 213.6)^{\mathrm{cd}}$ & $\begin{array}{l}1835.6 \\
( \pm 245.8)^{\text {bd }}\end{array}$ & $<0.0001$ \\
\hline Missing & $25(14.0 \%)$ & $15(12.0 \%)$ & $21(12.6 \%)$ & $20(14.5 \%)$ & \\
\hline Energy expenditure (kcal/kg/d) & $32.5( \pm 2.9)$ & $32.8( \pm 2.6)$ & $32.7( \pm 1.7)$ & $32.5( \pm 1.7)$ & 0.56 \\
\hline Missing & $26(14.5 \%)$ & $6(4.8 \%)$ & $17(10.2 \%)$ & $6(4.3 \%)$ & \\
\hline \multicolumn{6}{|l|}{ Genotype } \\
\hline Low-carb & $57(31.8 \%)$ & $40(32.0 \%)$ & $40(24.0 \%)$ & $43(31.2 \%)$ & 0.15 \\
\hline Low-fat & $69(38.5 \%)$ & $45(36.0 \%)$ & $75(44.9 \%)$ & $55(39.9 \%)$ & \\
\hline Neither & $40(22.3 \%)$ & $36(28.8 \%)$ & $42(25.1 \%)$ & $28(20.3 \%)$ & \\
\hline None & $3(1.7 \%)$ & $0(0.0 \%)$ & $6(3.6 \%)$ & $6(4.3 \%)$ & \\
\hline Missing & $10(5.6 \%)$ & $4(3.2 \%)$ & $4(2.4 \%)$ & $6(4.3 \%)$ & \\
\hline Smoking status & & & & & 0.22 \\
\hline Non-Smoker & $137(76.5 \%)$ & $92(73.6 \%)$ & $137(82.0 \%)$ & $101(73.2 \%)$ & \\
\hline Current smoker & $0(0.0 \%)$ & $0(0.0 \%)$ & $0(0.0 \%)$ & $0(0.0 \%)$ & \\
\hline Past smoker & $42(23.5 \%)$ & $33(26.4 \%)$ & $30(18.0 \%)$ & $37(26.8 \%)$ & \\
\hline
\end{tabular}

AI American Indian, AN Alaskan Native, PI Pacific Islander.

${ }^{1}$ Shared superscript symbols (a, b, c, d, e) indicate significant between-group differences (Kruskal-Wallis test for continuous variables, e.g., age, and Fisher's exact test for categorical variables, e.g., race). 
line with their physiological propensity to store fat in visceral rather than subcutaneous depots.

There was no significant between-group difference in baseline percent macronutrient intake between groups with the exception of a modest $1 \%$ greater intake of protein among HLC men compared to HLF men $(P=0.047)$ (Table 2). At all postrandomization time points, HLC women and men reported lower percent carbohydrate intake than HLF, and HLF women and men reported lower percent fat intake than HLC $(P<0.001)$.

\section{Changes in weight, body fat, and lean mass}

After adjustment for differences in baseline weight and body fat percentage, we observed significant differences in 12-month weight loss $[F(3,1221.14)=5.95, P<0.001]$ and loss of lean mass $[F(3,816.46)=9.21, P<0.001]$. Among men, HLC induced significantly greater weight loss than HLF $[-2.98 \mathrm{~kg}(-4.47,-1.50) ; P<0.001]$. This was also observed for fat mass $[-1.51(-2.79,-0.23) ; P=0.02]$, and, for lean mass $[-1.33(-1.97,-0.68) ; P<0.001]$ (Fig. 1). In contrast, there was no differential effect by diet type on weight loss, and loss of fat and lean mass among women, who achieved similar changes on HLC and HLF. In addition, HLC men lost significantly more weight $[-2.32$ $(-3.67,-0.97) ; P<0.001]$ and lean mass $[-1.42(-2.01$, $-0.84) ; P<0.001]$ than HLC women. The loss of weight, fat mass, and lean mass were not significantly different between HLF women and HLF men.

\section{Diet adherence}

Though the overall differences in adherence by diet-sex group were not statistically significant $[F(3,553)=2.06$, $p=0.10]$ (Fig. 2), HLC men and HLC women were the most and least adherent of all groups, respectively; in pairwise analyses this difference reached modest significance [WASA difference: $0.25(0.04,0.46) ; P=0.02$ ]

\section{Associations between diet adherence and changes in weight, body fat, and lean mass}

For all groups diet adherence correlated significantly with 12-month changes in percent weight (HLC women, $\mathrm{rs}=$ $-0.36, P<0.001$; HLC men, $\mathrm{rs}=-0.57, P<0.001$; HLF women, $\mathrm{rs}=-0.32, P<0.001$; HLF men, $\mathrm{rs}=-0.36$, $P<0.001)$ and fat mass with the exception of HLF men (HLC women, $\mathrm{rs}=-0.29, P=0.002$; HLC men, $\mathrm{rs}=$ $-0.60, P<0.001$; HLF women, $\mathrm{rs}=-0.27, P=0.01$; HLF men, rs $=-0.22, P=0.066$ ) (Supplementary Figs. S1 and S2). Diet adherence also correlated significantly with 12 month percent changes in lean mass for all the groups with the exception of HLF women bordering on the
$P<0.05$ significance cutoff (HLC women, $\mathrm{rs}=-0.23$, $P=0.014$; HLC men, $\mathrm{rs}=-0.45, P<0.001$; HLF women, rs $=-0.20, P=0.054$; HLF men, $\mathrm{rs}=-0.29, P=0.016$ ) (Supplementary Fig. S3). Weight loss estimates within group remained similar after adjusting for adherence, suggesting it was not a mediator (Supplementary Table S2).

\section{Attitudes toward dietary fats and carbohydrates}

The food choice questionnaire data were used to explore whether the observed differences in diet adherence between women and men might reflect different attitudes toward dietary fats and carbohydrates (Table 3). At baseline, a significantly greater proportion of women vs. men (58\% vs. $39 \%, p \leq 0.0001)$ rated the importance of consuming LF foods as very or moderately important. Also assessed at baseline were attitudes toward foods particularly high in refined carbohydrates (e.g., bread, pasta, rice), which were restricted on both HLC and HLF. A significantly greater proportion of women vs. men $(27 \%$ vs. $18 \%, p=0.0009)$ reported a tendency to avoid these foods always, very often or often.

\section{Discussion}

By factoring sex into this secondary analysis of the DIETFITS trial, we identified a significant interaction of sex on the effects of a HLC vs. HLF diet on weight loss success, that was not addressed in the parent analysis, when data on women and men were combined [27]. After accounting for differences in baseline weight and body fat percentage, we observed a significant effect of diet intervention type on weight, fat, and lean loss among men but not among women. Men lost significantly more weight, fat mass, and lean mass on HLC diet vs. HLF, whereas women achieved similar losses on HLC vs. HLF. In addition, within the HLC group, men lost significantly more weight than women, which was not true for HLF.

Behavioral differences in diet adherence may explain the greater weight loss success of men on HLC. We found that men were significantly more adherent to HLC than women. Interestingly, prior to randomization, the women expressed a significantly greater preference for LF foods than the men, which might have made it more difficult for the former to adhere to the higher fat content of HLC. In line with an overall greater attitude to weight control, the women also reported a significantly higher tendency to avoid foods high in refined carbohydrates (e.g., bread, pasta, rice). However, since these foods were restricted on both HLC and HLF, this attitude should not have differentially affected diet adherence to HLC or HLF among women. These findings 
Table 2 Macronutrient intake by subgroup and time point.

\begin{tabular}{|c|c|c|c|c|c|}
\hline & HLC women & HLC men & HLF women & HLF men ${ }^{1}$ & $P$ value 2 \\
\hline \multicolumn{6}{|c|}{ Total calories, mean (SD) } \\
\hline Baseline & $2096( \pm 615)^{\mathrm{ab}}$ & $2405( \pm 665)^{\mathrm{ac}}$ & $1985( \pm 605)^{\mathrm{cd}}$ & $2345( \pm 729)^{\mathrm{bd}}$ & $<0.0001$ \\
\hline 3 months & $1492( \pm 440)^{\mathrm{ab}}$ & $1708( \pm 512)^{\mathrm{ac}}$ & $1410( \pm 380)^{\mathrm{cd}}$ & $1648( \pm 514)^{\mathrm{bd}}$ & $<0.0001$ \\
\hline 6 months & $1508( \pm 429)^{\mathrm{ab}}$ & $1779( \pm 605)^{\mathrm{ac}}$ & $1429( \pm 421)^{\mathrm{cd}}$ & $1872( \pm 651)^{\mathrm{bd}}$ & $<0.0001$ \\
\hline 12 months & $1576( \pm 442)^{\mathrm{ab}}$ & $1871( \pm 483)^{\mathrm{ac}}$ & $1587( \pm 452)^{\mathrm{cd}}$ & $1884( \pm 551)^{\mathrm{bd}}$ & $<0.0001$ \\
\hline \multicolumn{6}{|c|}{ Total carb, mean (SD), g } \\
\hline Baseline & $237( \pm 76)^{\mathrm{a}}$ & $261( \pm 80)^{\mathrm{ab}}$ & $228( \pm 76)^{\mathrm{bc}}$ & $259( \pm 95)^{\mathrm{c}}$ & 0.001 \\
\hline 3 months & $99( \pm 58)^{\mathrm{ab}}$ & $93( \pm 55)^{\mathrm{cd}}$ & $189( \pm 61)^{\text {ace }}$ & $226( \pm 78)^{\text {bde }}$ & $<0.0001$ \\
\hline 6 months & $108( \pm 56)^{\mathrm{ab}}$ & $120( \pm 75)^{\mathrm{cd}}$ & $185( \pm 63)^{\text {ace }}$ & $244( \pm 91)^{\text {bde }}$ & $<0.0001$ \\
\hline 12 months & $129( \pm 62)^{\mathrm{ab}}$ & $137( \pm 66)^{\mathrm{cd}}$ & $195( \pm 68)^{\text {ace }}$ & $236( \pm 78)^{\text {bde }}$ & $<0.0001$ \\
\hline \multicolumn{6}{|c|}{ Total fat, mean (SD), $\mathrm{g}$} \\
\hline Baseline & $88( \pm 32)^{\mathrm{a}}$ & $100( \pm 35)^{\mathrm{ab}}$ & $79( \pm 29)^{\mathrm{bc}}$ & $96( \pm 39)^{\mathrm{c}}$ & $<0.0001$ \\
\hline 3 months & $83( \pm 27)^{\mathrm{ab}}$ & $98( \pm 37)^{\mathrm{cd}}$ & $41( \pm 18)^{\mathrm{ac}}$ & $44( \pm 23)^{\mathrm{bd}}$ & $<0.0001$ \\
\hline 6 months & $81( \pm 27)^{\mathrm{ab}}$ & $95( \pm 35)^{\mathrm{cd}}$ & $45( \pm 21)^{\text {ace }}$ & $57( \pm 32)^{\text {bde }}$ & $<0.0001$ \\
\hline 12 months & $79( \pm 27)^{\mathrm{ab}}$ & $96( \pm 32)^{\mathrm{cd}}$ & $54( \pm 22)^{\text {ace }}$ & $61( \pm 28)^{\text {bde }}$ & $<0.0001$ \\
\hline \multicolumn{6}{|c|}{ Total protein, mean (SD), $\mathrm{g}$} \\
\hline Baseline & $86( \pm 25)^{\mathrm{ab}}$ & $104( \pm 30)^{\mathrm{ac}}$ & $84( \pm 25)^{\mathrm{cd}}$ & $102( \pm 31)^{\mathrm{bd}}$ & $<0.0001$ \\
\hline 3 months & $87( \pm 24)^{\mathrm{ab}}$ & $110( \pm 38)^{\mathrm{acd}}$ & $73( \pm 20)^{\text {bce }}$ & $88( \pm 32)^{\mathrm{de}}$ & $<0.0001$ \\
\hline 6 months & $85( \pm 24)^{\mathrm{ab}}$ & $106( \pm 34)^{\mathrm{acd}}$ & $72( \pm 23)^{\text {bce }}$ & $94( \pm 32)^{\mathrm{de}}$ & $<0.0001$ \\
\hline 12 months & $85( \pm 22)^{\mathrm{a}}$ & $106( \pm 35)^{\mathrm{ab}}$ & $78( \pm 25)^{\mathrm{bc}}$ & $93( \pm 27)^{\mathrm{c}}$ & $<0.0001$ \\
\hline \multicolumn{6}{|c|}{ Total carb, mean (SD), \% } \\
\hline Baseline & $45( \pm 7)$ & $43( \pm 7)$ & $45( \pm 7)$ & $44( \pm 10)$ & 0.15 \\
\hline 3 months & $25( \pm 11)^{\mathrm{ab}}$ & $21( \pm 11)^{\mathrm{cd}}$ & $52( \pm 10)^{\mathrm{ac}}$ & $53( \pm 11)^{\mathrm{bd}}$ & $<0.0001$ \\
\hline 6 months & $27( \pm 10)^{\mathrm{ab}}$ & $26( \pm 11)^{\mathrm{cd}}$ & $51( \pm 10)^{\mathrm{ac}}$ & $51( \pm 11)^{\mathrm{bd}}$ & $<0.0001$ \\
\hline 12 months & $31( \pm 11)^{\mathrm{ab}}$ & $28( \pm 11)^{\mathrm{cd}}$ & $48( \pm 10)^{\mathrm{ac}}$ & $49( \pm 9)^{\mathrm{bd}}$ & $<0.0001$ \\
\hline \multicolumn{6}{|c|}{ Total fat, mean (SD), \% } \\
\hline Baseline & $36( \pm 6)$ & $36( \pm 6)$ & $35( \pm 6)$ & $35( \pm 7)$ & 0.095 \\
\hline 3 months & $49( \pm 9)^{\mathrm{ab}}$ & $50( \pm 9)^{\mathrm{cd}}$ & $25( \pm 8)^{\mathrm{ac}}$ & $23( \pm 8)^{\mathrm{bd}}$ & $<0.0001$ \\
\hline 6 months & $47( \pm 9)^{\mathrm{ab}}$ & $46( \pm 9)^{\mathrm{cd}}$ & $27( \pm 8)^{\mathrm{ac}}$ & $26( \pm 9)^{\mathrm{bd}}$ & $<0.0001$ \\
\hline 12 months & $44( \pm 9)^{\mathrm{ab}}$ & $45( \pm 9)^{\mathrm{cd}}$ & $30( \pm 8)^{\mathrm{ac}}$ & $28( \pm 7)^{\mathrm{bd}}$ & $<0.0001$ \\
\hline \multicolumn{6}{|c|}{ Total protein, mean (SD), \% } \\
\hline Baseline & $17( \pm 4)^{\mathrm{a}}$ & $18( \pm 3)^{\mathrm{a}}$ & $18( \pm 4)$ & $18( \pm 5)$ & 0.047 \\
\hline 3 months & $25( \pm 6)^{\mathrm{ab}}$ & $27( \pm 7)^{\mathrm{cd}}$ & $21( \pm 5)^{\mathrm{ac}}$ & $22( \pm 7)^{\mathrm{bd}}$ & $<0.0001$ \\
\hline 6 months & $24( \pm 6)^{\mathrm{ab}}$ & $25( \pm 7)^{\mathrm{cd}}$ & $21( \pm 6)^{\mathrm{ac}}$ & $21( \pm 7)^{\mathrm{bd}}$ & $<0.0001$ \\
\hline 12 months & $23( \pm 6)$ & $23( \pm 6)^{b c}$ & $21( \pm 6)^{b}$ & $21( \pm 6)^{c}$ & 0.0007 \\
\hline
\end{tabular}

${ }^{a}$ Dietary data were available at baseline and 3, 6, and 12 months, respectively, as follows: HLC women, $n=$ 179, 162, 146, 132; HLC men, $n=125,113,105$, 92; HLF women, $n=166,153$, 134, 127; HLF men, $n=$ 138, 121, 106, 98.

${ }^{\mathrm{b}}$ From Kruskal-Wallis omnibus test; shared superscript symbols (a, b, c, d, e) indicate significant betweengroup differences (Dunn's test, Bonferroni adjusted $p<0.05$ ). are consistent with several large population studies indicating that women express a greater preference for LF products and a greater concern toward high-fat foods than men [12, 31-41]. Sociologists suggest that women may be concerned about eating dietary fats due to a gender stereotype that pressure them to be slim [42-44] and avoid foods perceived as "fattening" [45-47]—a stereotype that is reflected and reinforced by gender marketing of LF products to women [48, 49].
Although adherence was significantly correlated with weight loss for all groups, this association was of greater magnitude for HLC than HLF. This is in agreement with a previous study that observed a significant association between greater adherence to a low carb diet and greater weight loss that was not observed for the low-fat diet [26]. The association between diet adherence and weight loss was also greater for men than for women, with HLC men having the strongest correlation. 
Fig. 1 12-month changes in weight, fat mass, and lean mass by sex and diet, adjusted for baseline weight and baseline body fat percentage. Estimated between-group differences $(95 \% \mathrm{CI})$. Weight data were available at baseline and 3,6 , and 12 months, respectively, as follows: women on HLF, $n=167,151,131,120$; women on HLC, $n=179,155$, 144, 131; men on HLF, $n=138$, 119, 97, 96; and men on HLC, $n=125,108,95,89$. Fat mass and lean mass data were available at baseline, 6 , 12 months, respectively, as follows (cohorts 2-5): women on HLF, $n=131,107,106$; women on HLC, $n=145,121$, 118; men on HLF, $n=97,75$, 79; men on HLC, $n=93,73,78$.

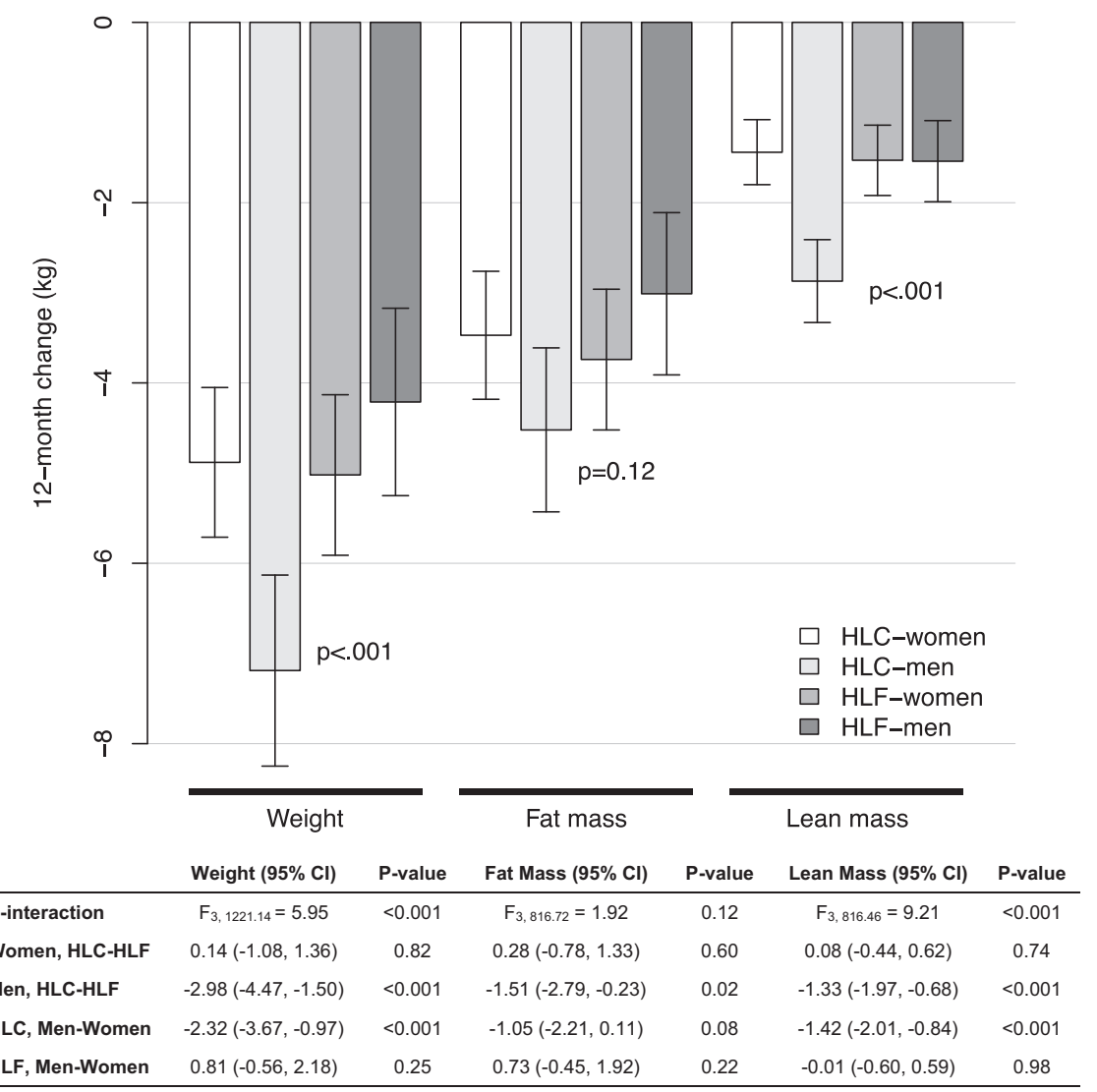

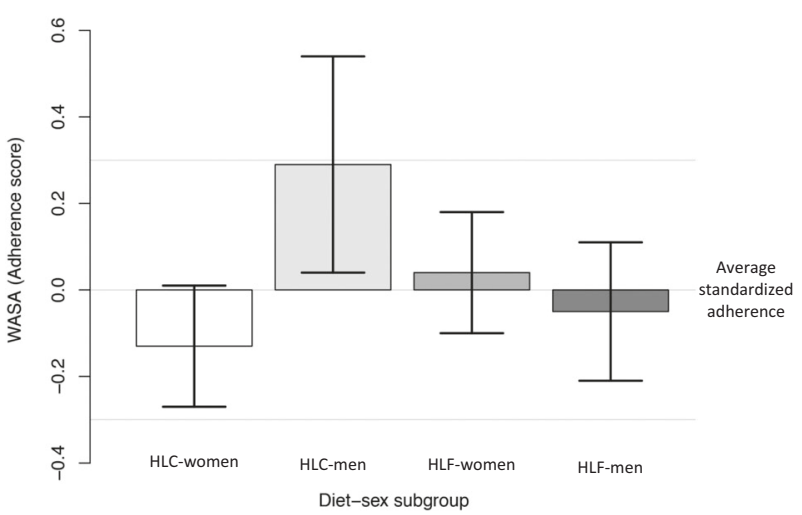

Fig. 2 Differences in 12-month diet adherence by sex and diet type. Estimated between-group differences in weight-adjusted standardized adherence (WASA) scores. Higher values indicate higher adherence relative to the average adherence across all groups.

Our findings are consistent with previous reports that a low carb diet may produce more effective weight loss in men than women $[9,16,17]$. Volek et al. reported that men $(n=13)$ lost significantly more absolute weight and fat on a very low-carbohydrate diet (VLCD) compared with a LF diet, whereas VLCD was less effective for women $(n=15)$ [9]. However, comparisons between women and men on either VLCD or LF were not made, probably due to the
Table 3 Food attitudes at baseline, by sex.

\begin{tabular}{llll}
\hline & $\begin{array}{l}\text { Female } \\
n=346\end{array}$ & $\begin{array}{l}\text { Male } \\
n=263\end{array}$ & $P$ value $^{\mathrm{a}}$ \\
\hline Low-fat food on a typical day & & & \\
$\quad$ Very important & $45(13.0 \%)$ & $23(8.7 \%)$ & $<0.0001$ \\
Moderately important & $157(45.4 \%)$ & $79(30.0 \%)$ & \\
A little important & $107(30.9 \%)$ & $115(43.7 \%)$ & \\
Not at all & $32(9.2 \%)$ & $43(16.3 \%)$ & \\
Missing & $5(1.4 \%)$ & $3(1.1 \%)$ & \\
Avoiding refined & & & \\
carbohydrates & & & \\
Always & $4(1.2 \%)$ & $3(1.1 \%)$ & 0.0009 \\
Very often & $43(12.4 \%)$ & $21(8.0 \%)$ & \\
Often & $48(13.9 \%)$ & $24(9.1 \%)$ & \\
Sometimes & $103(29.8 \%)$ & $55(20.9 \%)$ & \\
Rarely & $89(25.7 \%)$ & $97(36.9 \%)$ & \\
Never & $54(15.6 \%)$ & $59(22.4 \%)$ & \\
Missing & $5(1.4 \%)$ & $4(1.5 \%)$ &
\end{tabular}

${ }^{\text {a}}$ Fisher's exact test.

small sample size. Similarly, in a study of 33 men and 45 women on four dietary regimens (Atkins, Slim-Fast, Weight-Watchers, and Rosemary Conley's Diet), Millward et al. reported that men in the Atkins group had a 
significantly greater, albeit transient, reduction in body weight and fat compared to all other groups. However, baseline weight was not accounted. In a 2-year study of 322 moderately obese participants (men: $86 \%$ ), Shai et al. found that a low-carbohydrate diet was more effective for men whereas women tended to lose more weight on a Mediterranean diet [16]. The current study builds on these previous studies and identifies a significant effect modification of sex on both weight loss and loss of lean mass. Not only did HLC men lost significantly more weight than HLF men, they also lost both more fat and lean mass, with the lean mass differences being more statistically significant than the fat mass differences.

Our analysis has several important strengths. First, we used data derived from a relatively large RCT with sample sizes in each of the four diet-sex groups of $n=125$ to 179 , good retention $(\sim 70 \%$ for all groups), and a sufficient duration (12 months) to evaluate long-term effects of two diets with substantially different macronutrient composition. In addition, we designed a novel WASA score to enable comparisons across diet and sex groups.

This study has also several limitations. First, this secondary analysis was not set out in the original study protocol, and hence participants were not stratified by sex prior to randomization. Nonetheless, randomization yielded similar baseline characteristics within each sex. A second major limitation is that we did not analyze other sex-related factors, such as genotype, hormones, MetS, or psychosocial factors that might affect either diet adherence or weight loss response. Third, although our WASA score was specifically designed to enable comparisons across both sex and diet, this novel metric has not been validated. Therefore, as any comparison of data between groups with different means, ranges, and standard deviations, it should be interpreted with caution. Self-report of dietary intake might also have affected the assessment of adherence. For example, individuals who underreported their intake-as is common in diet assessment [50] - might have been misclassified with an inaccurate WASA. Finally, as in most weight loss diet studies, complete data were missing for a subset of participants at both baseline and 12 months.

In conclusion, we found modest but significantly different losses of weight, fat mass, and lean mass by diet-sex groups, with adherence being significantly correlated with weight loss in all groups. Women may find it easier to adhere to a LF diet than to a low-carb diet due to gender norms and marketing strategies that make LF products more appealing and accessible in the marketplace. To increase low-carb adherence, and thus increase opportunity for success, healthcare providers may want to inform their female patients about the comparable effectiveness of a low-carb diet to LF diet, as such beliefs have a strong influence of food attitudes [51-54]. Our findings also beg the question of whether heterogeneous treatment effects (HTEs) due to sex differences could explain some of the variability in weight loss outcome in response to different diet interventions. Since any subgroup analysis lessens power and therefore the ability to detect effects, this question should be tested in randomized clinical trials with large sample sizes and stratification by sex prior to randomization. Unbiased estimation of sex-related HTEs on diet response from such a trial could provide better clinical evidence for the implementation of personalized weight loss strategies based on sex differences. As precision medicine grows steadily, analysis of sex differences should become a priority in comparative effectiveness trials of different diets designed for healthy weight loss $[55,56]$.

Acknowledgements We would like to thank Jin Long at the Quantitative Sciences Unit, Stanford University School of Medicine, for providing critical input for the statistical analysis. We also acknowledge the 346 women and 263 men participants in this study without whom this investigation would not have been possible. LA was supported by the European Union's Horizon 2020 Research and Innovation Program under grant agreement no. 701944. CG was supported by grant 1R01DK091831 from the National Institute of Diabetes and Digestive and Kidney Diseases, and NIH National Center for Advancing Translational Science Clinical and Translational Science Award (UL1TR001085). The NIH IRACDA Postdoctoral Fellowship (1 K12 GM088033) supported Dr LCO at Stanford University.

\section{Compliance with ethical standards}

Conflict of interest The authors declare that they have no conflict of interest.

Publisher's note Springer Nature remains neutral with regard to jurisdictional claims in published maps and institutional affiliations.

Open Access This article is licensed under a Creative Commons Attribution 4.0 International License, which permits use, sharing, adaptation, distribution and reproduction in any medium or format, as long as you give appropriate credit to the original author(s) and the source, provide a link to the Creative Commons license, and indicate if changes were made. The images or other third party material in this article are included in the article's Creative Commons license, unless indicated otherwise in a credit line to the material. If material is not included in the article's Creative Commons license and your intended use is not permitted by statutory regulation or exceeds the permitted use, you will need to obtain permission directly from the copyright holder. To view a copy of this license, visit http://creativecommons. org/licenses/by/4.0/.

\section{References}

1. Clayton JA. Studying both sexes: a guiding principle for biomedicine. FASEB J. 2016;30:519-24.

2. Tannenbaum C, Schwarz JM, Clayton JA, de Vries GJ, Sullivan C. Evaluating sex as a biological variable in preclinical research: the devil in the details. Biol Sex Diff. 2016;7:13.

3. Clayton JA. Applying the new SABV (sex as a biological variable) policy to research and clinical care. Physiol Behav. 2018;187:2-5. 
4. Greenberg I, Stampfer MJ, Schwarzfuchs D, Shai I. Adherence and success in long-term weight loss diets: the dietary intervention randomized controlled trial (DIRECT). J Am College Nutr. 2009;28:159-68.

5. Wu BN, O’Sullivan AJ. Sex differences in energy metabolism need to be considered with lifestyle modifications in humans. J Nutr Metabol. 2011;2011:391809.

6. Cunningham JJ. Body composition as a determinant of energy expenditure: a synthetic review and a proposed general prediction equation. Am J Clin Nutr. 1991;54:963-9.

7. Jackson AS, Stanforth PR, Gagnon J, Rankinen T, Leon AS, Rao DC, et al. The effect of sex, age and race on estimating percentage body fat from body mass index: The Heritage Family Study. Int J Obes Relat Metabol Disord. 2002;26:789-96.

8. Hamdy O, Porramatikul S, Al-Ozairi E. Metabolic obesity: the paradox between visceral and subcutaneous fat. Curr Diab Rev. 2006;2:367-73.

9. Volek J, Sharman M, Gomez A, Judelson D, Rubin M, Watson G, et al. Comparison of energy-restricted very low-carbohydrate and low-fat diets on weight loss and body composition in overweight men and women. Nutr Metabol. 2004;1:13.

10. Bellisle F, Monneuse MO, Steptoe A, Wardle J. Weight concerns and eating patterns: a survey of university students in Europe. Int $\mathbf{J}$ Obes Relat Metabol Disord. 1995;19:723-30.

11. Wardle J, Griffith J. Socioeconomic status and weight control practices in British adults. J Epidemiol Commun Health. 2001;55:185-90.

12. Arganini C, Saba A, Comitato R, Virgili F, Turrini A. Gender differences in food choice and dietary intake in modern western societies. Public Health IntechOpen; 2012.

13. Williams RL, Wood LG, Collins CE, Callister R. Effectiveness of weight loss interventions - is there a difference between men and women: a systematic review. Obe Rev. 2015;16:171-86.

14. Robertson C, Avenell A, Boachie C, Stewart F, Archibald D, Douglas F, et al. Should weight loss and maintenance programmes be designed differently for men? A systematic review of long-term randomised controlled trials presenting data for men and women: The ROMEO project. Obe Res Clin Pract. 2016;10:70-84.

15. Luscombe-Marsh ND, Noakes M, Wittert GA, Keogh JB, Foster P, Clifton PM. Carbohydrate-restricted diets high in either monounsaturated fat or protein are equally effective at promoting fat loss and improving blood lipids. Am J Clin Nutr. 2005;81:762-72.

16. Shai I, Schwarzfuchs D, Henkin Y, Shahar DR, Witkow S, Greenberg I, et al. Weight loss with a low-carbohydrate, Mediterranean, or low-fat diet. N. Eng J Med. 2008;359:229-41.

17. Millward DJ, Truby H, Fox KR, Livingstone MB, Macdonald IA, Tothill P. Sex differences in the composition of weight gain and loss in overweight and obese adults. Br J Nutr. 2014;111:933-43.

18. Lopez-Legarrea P, de la Iglesia R, Abete I, Bondia-Pons I, NavasCarretero S, Forga L, et al. Short-term role of the dietary total antioxidant capacity in two hypocaloric regimes on obese with metabolic syndrome symptoms: the RESMENA randomized controlled trial. Nutr Metabol. 2013;10:22.

19. Stocks T, Ängquist L, Banasik K, Harder MN, Taylor MA, Hager $\mathrm{J}$, et al. TFAP2B influences the effect of dietary fat on weight loss under energy restriction. PLOS One. 2012;7:e43212.

20. Ma W, Huang T, Wang M, Zheng Y, Wang T, Heianza Y, et al. Two-year changes in circulating adiponectin, ectopic fat distribution and body composition in response to weight-loss diets: the POUNDS Lost Trial. Int J Obes. 2016;40:1723-9.

21. Janssen I, Ross R. Effects of sex on the change in visceral, subcutaneous adipose tissue and skeletal muscle in response to weight loss. Int J Obes. 1999;23:1035.

22. St-Onge MP, Salinardi T, Herron-Rubin K, Black RM. A weightloss diet including coffee-derived mannooligosaccharides enhances adipose tissue loss in overweight men but not women. Obesity. 2012;20:343-8.

23. Sasakabe T, Haimoto H, Umegaki H, Wakai K. Effects of a moderate low-carbohydrate diet on preferential abdominal fat loss and cardiovascular risk factors in patients with type 2 diabetes. Diab Metabol Synd Obes. 2011;4:167-74.

24. Evans EM, Mojtahedi MC, Thorpe MP, Valentine RJ, KrisEtherton PM, Layman DK. Effects of protein intake and gender on body composition changes: a randomized clinical weight loss trial. Nutr Metabol. 2012;9:55.

25. Farnsworth E, Luscombe ND, Noakes M, Wittert G, Argyiou E, Clifton PM. Effect of a high-protein, energy-restricted diet on body composition, glycemic control, and lipid concentrations in overweight and obese hyperinsulinemic men and women. Am J Clin Nutr. 2003;78:31-9.

26. Hu T, Yao L, Reynolds K, Niu T, Li S, Whelton PK, et al. Adherence to low-carbohydrate and low-fat diets in relation to weight loss and cardiovascular risk factors. Obes Sci Pract. 2016;2:24-31.

27. Gardner CD, Trepanowski JF, Del Gobbo LC, et al. Effect of lowfat vs low-carbohydrate diet on 12-month weight loss in overweight adults and the association with genotype pattern or insulin secretion: the dietfits randomized clinical trial. JAMA. 2018;319:667-79.

28. Stanton MV, Robinson JL, Kirkpatrick SM, Farzinkhou S, Avery EC, Rigdon J, et al. DIETFITS study (diet intervention examining the factors interacting with treatment success) - Study design and methods. Contemp Clin Trials. 2017;53:151-61.

29. Feskanich D, Sielaff BH, Chong K, Buzzard IM. Computerized collection and analysis of dietary intake information. Comput Methods Prog Biomed. 1989;30:47-57.

30. Team RCR. A language and environment for statistical computing. Vienna, Austria: R Foundation for Statistical Computing; 2017.

31. Rozin P, Fischler C, Imada S, Sarubin A, Wrzesniewski A. Attitudes to food and the role of food in life in the U.S.A., Japan, Flemish Belgium and France: possible implications for the diethealth debate. Appetite. 1999;33:163-80.

32. Dowd D, Peel JC. Dietary fat reduction practices by college males and females. College Student. Journal. 1992;26:231-6.

33. Wardle J, Beales S. Restraint, body image and food attitudes in children from 12 to 18 years. Appetite. 1986;7:209-17.

34. Bushman BJ. Effects of warning and information labels on consumption of full-fat, reduced-fat, and no-fat products. J Appl Psychol. 1998;83:97-101.

35. Wardle J, Haase AM, Steptoe A, Nillapun M, Jonwutiwes K, Bellisle F. Gender differences in food choice: the contribution of health beliefs and dieting. Ann Behav Med. 2004;27:107-16.

36. Fulkerson JA, French SA, Story M. Adolescents' attitudes about and consumption of low-fat foods: associations with sex and weight-control behaviors. J Am Diet Assoc. 2004;104:233-7.

37. Baghurst KI, Baghurst PA, Record SJ. Demographic and dietary profiles of high and low fat consumers in Australia. J Epidemiol Commun Health. 1994;48:26-32.

38. Liebman M, Cameron BA, Carson DK, Brown DM, Meyer SS. Dietary fat reduction behaviors in college students: relationship to dieting status, gender and key psychosocial variables. Appetite. 2001;36:51-6.

39. Hunt MK, Stoddard AM, Glanz K, Hebert JR, Probart C, Sorensen $\mathrm{G}$, et al. Measures of food choice behavior related to intervention messages in worksite health promotion. J Nutr Educ. 1997;29:3-11.

40. Margetts BM, Martinez JA, Saba A, Holm L, Kearney M, Moles A. Definitions of 'healthy' eating: a pan-EU survey of consumer attitudes to food, nutrition and health. Eur J Clin Nutr. 1997;51: S23-9. 
41. Block G, Rosenberger WF, Patterson BH. Calories, fat and cholesterol: intake patterns in the US population by race, sex and age. Am J Public Health. 1988;78:1150-5.

42. Shepherd R, Towler G. Nutrition knowledge, attitudes and fat intake: application of the theory of reasoned action. J Hum Nutr Diet. 2007;20:159-69.

43. La Berge AF. How the ideology of low fat conquered America. J Hist Med Allied Sci. 2008;63:139-77.

44. Stearns P. Fat History: Bodies and Beauty in the Modern West. New York: NYU Press; 2002.

45. Sobal J, Nicolopoulos V, Lee J. Attitudes about overweight and dating among secondary school students. Int J Obes Relat Metabol Disord. 1995; 19:376-81.

46. John G, Lauren W. The sexual division of dieting: women's voices. Sociol Rev. 1996;44:630-47.

47. Lowe MA. Looking good: college women and body image, 18751930. Gender relations and the American Experience. Baltimore: Johns Hopkins University Press; 2003.

48. Parkin KJ. Food is love: food advertising and gender roles in modern America. Philadelphia: University of Pennsylvania Press; 2006.

49. Bordo S. Unbearable weight: feminism, western culture and the body. Berkeley, CA: University of California Press; 1993.
50. Weber JL, Reid PM, Greaves KA, DeLany JP, Stanford VA, Going SB, et al. Validity of self-reported energy intake in lean and obese young women, using two nutrient databases, compared with total energy expenditure assessed by doubly labeled water. Eur J Clin Nutr. 2001;55:940-50.

51. Shepherd R, Sparks P. Measurement of food preferences. In: MacFie H, Thomson DMH, editors. Modelling food choice. Boston, MA: Springer; 1994.

52. Ajzen I, Fishbein M. The influence of attitudes on behavior. The handbook of attitudes. Mahwah, NJ, US: Lawrence Erlbaum Associates Publishers; 2005. p. 173-221.

53. Eagly AH, Chaiken S. The psychology of attitudes. Orlando, FL, US: Harcourt Brace Jovanovich College Publishers; 1993. p. 794.

54. Ajzen I. Consumer attitudes and behavior: the theory of planned behavior applied to food consumption decisions. Italian Rev Agricultural Economics. 2015:121-38.

55. de Roos B, Brennan L. Personalised interventions-a precision approach for the next generation of dietary intervention studies. Nutrients. 2017;9:847.

56. Segarra I, Modamio P, Fernández C, Mariño EL. Sex-divergent clinical outcomes and precision. Medicine. 2017;8:488. 